\title{
Comparison of Flatfoot on the Activities of Medial and Lateral Plantar Flexor Muscle During Heel Rising
}

\author{
Hui-won Jung ${ }^{1}$, PT, MSc, Won-gyu Yoo², PT, PhD \\ ${ }^{1}$ Department of Physical Therapy, The Graduate School, Inje University, ${ }^{2}$ Department of Physical Therapy, College of Health Care Medical \\ Science and Engineering, Inje University, Gimhae, Korea
}

\author{
Article Info \\ Received January 15, 2021 \\ Revised April 8, 2021 \\ Accepted April 20, 2021 \\ Corresponding Author \\ Won-gyu Yoo \\ E-mail:won7y@inje.ac.kr \\ https://orcid.org/0000-0001-6200-9674
}

\section{Key Words}

Bipedal heel-rise test

Electromyography

Flat feet

Gastrocnemius muscles

Plantar flexors

Navicular drop test
Background: Flat feet can be identified by assessing the collapse of the medial longitudinal arch (MLA) and these conditions can trigger epidemiological changes in the feet. Many of previous studies compared the muscle activity of lower body in terms of intervention and dynamics to treat the structural defect of flat feet. However, few studies have investigated or analyzed the muscle activity of gastrocnemius muscle in the subjects with flat feet.

Objects: This study investigated the differences in changes of medial and lateral plantar flexors in subjects with flat feet during bipedal heel-rise (BHR) task and analyzed the differences in muscle activity between two groups by measuring the electromyography (EMG) of abductor hallucis $(A H)$, tibialis anterior (TA), medial gastrocnemius (MG), lateral gastrocnemius (LG).

Methods: A total of Twenty one adult females participated in this experiment. Subjects were assigned to groups according to the navicular drop test. The task was applied to the leg, where the heel lifting action prevailed. The muscle activity of the medial and lateral feet plantar flexors was evaluated, and the \% maximum voluntary isometric contractions (\%MVIC) of these were compared.

Results: For the difference between groups the muscle activity (\%MVIC) of LG muscle was statistically significantly low in flat feet group compared to healthy feet group (flat feet: 64.57, healthy feet: $90.17 ; p<0.05)$.

Conclusion: The results of this study will contribute to identifying the muscle activities of medial and lateral feet plantar flexors among subjects with flat feet, which can cause abnormal epidemiological changes in the feet.

\section{INTRODUCTION}

The feet and ankle muscle, during standing or gait, change their structure in accordance with the movements, provide a momentum by using ground repulsion, and play an important role in weight bearing through the movement of the subtalar joint [1]. In particular, the medial longitudinal arch (MLA) consisting of navicular, talus, calcaneus, three cuboids and the first to third metatarsal bones [2] is related to shock absorption and force transmission in the standing position and during the gait [3]. The collapse of the MLA due to inadequate support of the structures in the weight-bearing position, however, may cause ecessive pronation of the feet, resulting in damage in lower body [4]. According to Subotnick [5], 60\% of the population has normal arches, 20\% have undulating or high ones, and the remaining $20 \%$ have flat feet or low feet arch.

The flat feet refers to a lack of ability to accept and disperse weight by the overstretching and weakening of ligament and plantar fascia [2]. The cause of flat feet is not yet clear although but it is known as a deformation in which the MLA is collapses or disappears completely due to the lateral blurring of the back feet and the inward shift of weight [6] and The previous electromyography (EMG) study of plantar intrinsic muscles supporting the MLA also reported that the change or damage of these muscles have effect on the height and shape of MLA [3].

In flat feet, the lack of normal tension due to overstretching and weakening of connective tissue leads to active activity in the intrinsic and extrinsic muscles to compensate for such inability [2]. 
The flat feet, therefore, affect gait since it is an abnormal alignment of the feet and ankles, therefore, causes the changes in the input of the proprioceptive sense of the feet, the alignment of the lower body, and muscle activity [7].

The previous studies also showed that the activity of some muscles (tibialis posterior, tibialis anterior [TA], toe flexors, and calf) in flat feet increased due to the excessive pronation of the ankle joint during gait $[3,8-10]$. Other previous studies observing that the association between changes in feet posture and risk of injury of the lower leg may occur in abnormal muscle activity, in addition, showed that the flat feet rely on additional muscle support during gait, which requires more energy consumption and makes feet more tired compared to healthy feet [11].

The calf muscle, a posterior leg muscle, is commonly used to determine the muscle activity in subjects with flat feet since it is a powerful plantar flexor. In addition, the joint action of the plantar fascia and posterior leg muscles during gait plays an important role in maintaining the arch by pulling up the plantar fascia [12].

Riemann et al. [10] and Cibulka et al. [13] conducted a study of gastrocnemius muscle activation in three different positions during heel lift. The study was conducted based on the research hypothesis that the force line was changed due to the rotation of the lower extremities and the calf muscles would have different activity patterns.

As a result, when toe-out in both the weight-bearing and non-weight-bearing positions, the muscle activity of the medial gastrocnemius (MG)-muscle was higher than that of the lateral gastrocnemius (LG)-mucle. This could lead to the conclusion that the position of the toe induced more muscle activation of the MG-muscle than the LG-muscle.

Therefore, we will compare the difference in muscle activity of the medial and lateral plantar flexion muscles including the inner and LG-muscles of the flatfeet group and healthy feet group subjects during the bipedal heel-rise (BHR) task of flatfeet subjects causing abnormal alignment of the feet and ankles.

The hypothesis of this study was to establish that there would be a difference in which the muscle activity of the medial lateral outer plantar flexion muscles statistically decreased or increased during the BHR-task compared to the healthy feet group in the flat feet group. For the purpose, EMG of four medial and lateral plantar flexors were measured when the BHR- task was performed of flatfeet and healthy feet subjects, and to compare and analyze the difference in muscle activity of each group.

The purpose of this study is to provide basic information to aid in the development of a flat feet mediated method as an experiment to determine the difference between ankle muscle activity and people with flat, healthy feet in BHR.

To this end, when performing the BHR-task in flatfeet and healthy feet subjects, EMG of the four medial and lateral plantar flexors were measured, and the differences in muscle activity in each group were compared and analyzed.

\section{MATERIALS AND METHODS}

\section{Subjects}

The study recruited 30 adult females (15 flat-feet, 15 healthy feet) who agreed to participate voluntarily after hearing explanations about the purpose of the study and how to proceed. The minimum sample size required for t-test was calculated using $G^{*}$ power (University of Kiel, Kiel, Germany). The number of groups was 2, the effect size was 0.7 , the power was 0.80 , and the significance level $(\alpha)$ was 0.05 . As a result, 11 subjects were calculated for the experimental group and the control group. However, the experiment was conducted on a total of 30 adult females, considering the selection of subjects, application of the exclusion criteria, and dropouts. All participants in the study were provided with warm indoor temperatures in a pleasant laboratory environment, of which 21 adult females, excluding 6 who experienced ankle injury within the last six months, excluding 3 drop out and a total of 9 out-of-center females, participated.

The subjects were classified into flat-feeted group and healthy feet group by measuring the height of the MLA using the navicular drop test (NDT).

The criteria for selecting the target person are those who differ by more than $10 \mathrm{~mm}$ [14] and those who can walk and exercise independently, during the NDT. The exclusion criteria were set as those who had lower than $5 \mathrm{~mm}$ in the test, neurological history, congenital deformities in the body, musculoskeletal or joint disease history affected by walking and single feet scribe. This study was conducted after passing the deliberation process by INJE University's Institutional Committee (approval number: INJE 201805017). 


\section{Instrumentation}

\section{1) Collect and analyze surface EMG data}

In this study, data were collected and processed using 2EM (4D-MT, Relive, Gimhae, Korea) which is an equipment for measuring EMG of abductor hallucis (AH), TA, MG, and LG during the task of lifting the both heels. The surface EMG electrodes kept the distance between electrodes at $2 \mathrm{~cm}$ and were attached parallel to the muscle fiber direction. The sampling rate of the myocardial signal was set to $1,000 \mathrm{~Hz}$, the bandpass used 0 to $500 \mathrm{~Hz}$, and the EMG signal was treated and analyzed by the root mean square root mean square (RMS) [15].

In this study, for uniformity of the experiment, the EMG of the dominant lower extremity muscle was measured, and the position of electrode attachment of each muscle was determined by referring to existing studies.

The dominant lower limb was determined through a question of asking which feet he usually uses when kicking the ball. In order to measure the EMG of the $\mathrm{AH}$-muscle, the superficial EMG was attached to the muscle tendon at $1-2 \mathrm{~cm}$ behind the rough surface of the dorsum bone [16].

The TA-muscle was attached to the muscle tendon at $1 / 3$ of the calf bone and the inner ankle bone [17].

In addition, the MG-muscle is attached to the inner circumference of the gastrocnemius at $1 / 3$ of the knee, and the LG at $1 / 3$ of the gastrocnemius at the outer circumference of the knee [15]. Ground electrodes are attached to the center of the dominant leg malleolus.

Muscle activation of the AH, TA and MG, LG muscle was measured for 5 seconds of two repetitions during the two leg heel-up action.

The maximum voluntary isometric contractions (MVICs) of each muscle were measured based on a study by Kendall et al. [18] for normalization of muscle activity. In order to prevent skin contact between the tester and the target during MVIC measurement, insulation condition was created using a towel.

The MVIC of the AH-muscle was applying resistance to the inner face of the thumb toe with the other hand, by performing a MVIC in the opposite direction of the resistance applied.

The MVIC of the TA-muscle was measured by holding the subject's dominant distal calf in the position in which the subject sat directly, and then applying resistance in the direction of inversion-plantar flexion of the feet with the other hand, by performing a MVIC in the opposite direction of the resistance applied.

The MVIC of the MG and LG muscles was measured while the subject was prone to the table, and the maximum isometric contraction was performed when the tester held the subject's heel and applied resistance in the instep bending direction.

Muscle activity was repeated 2 for 5 seconds in the MVIC measurement of each muscle. The average 2-time root-power values for the middle 3 seconds, excluding the first and last 1 second of the measured RMS values for 5 seconds, were used as the normalized value (\%MVIC) [18].

\section{2) Navicular drop test}

In this study, the test was performed using an index card and a ruler.

Subjects were asked to wear shorts and participated in the experiment with bare feet. After being asked to sit comfortably in a chair with the hip and knee joints bent at 90 degrees, the feet are put down on the floor without weight support on a solid support surface. The examiner placed the ankle and subtalar joint in a neutral position and then performed a dominant leg side examination (Figure 1).

The examiner marked the rough surface of the medial navicular tuberosity on the side of the subject's dominant leg, and then marked the vertical distance to the floor on a thin and long index card without elasticity. When the test was per-
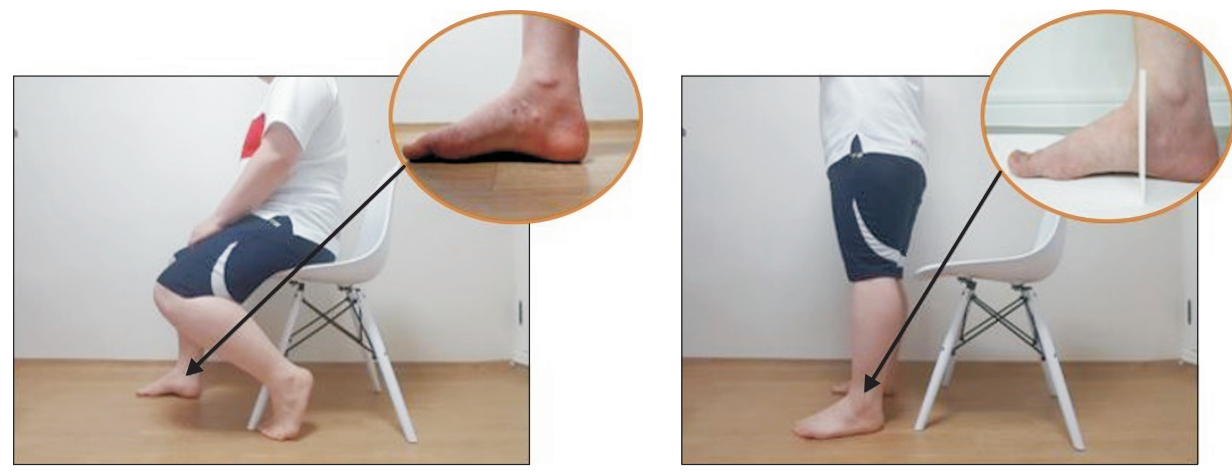

Figure 1. Navicular drop test. 
formed, the use of the upper limb was instructed to be excluded, and at the time of the tester's signal, the subject was asked to stand with the same weight on both feet while supporting the body weight.

After the subject was instructed to stand up completely from the chair at a comfortable speed, the examiner marked the marking on the rough surface of the medial navicular tuberosity on the subject's dominant leg side on the index card in the same way and recorded the vertical distance difference.

\section{3) $\mathrm{BHR}$}

Both the flat-feet and healthy-feet groups were examined the BHR-test, and the EMG only applied to the dominant leg (Figure 2). For the test, subjects were tested barefeet in shorts. The subjects were allowed to stand comfortably facing the wall without bending the knee joints and hip joints, and to support the wall using a small force that would allow them to bend their elbows and maintain balance with their fingers when the task began. Inspecting the dominant legs, the subjects were told to raise their heels to the maximum height to maintain balance between 5 and 15 seconds. To minimize discomfort and maximize the maximum heel height, the subject was repeated up to two times. It was also required to maintain the heel lifting position for 5 seconds to collect the subject's EMG data.

\section{4) Statistical analysis}

PASW ver. 18.0 (Formerly SPSS Statistics; IBM Co., Armonk,

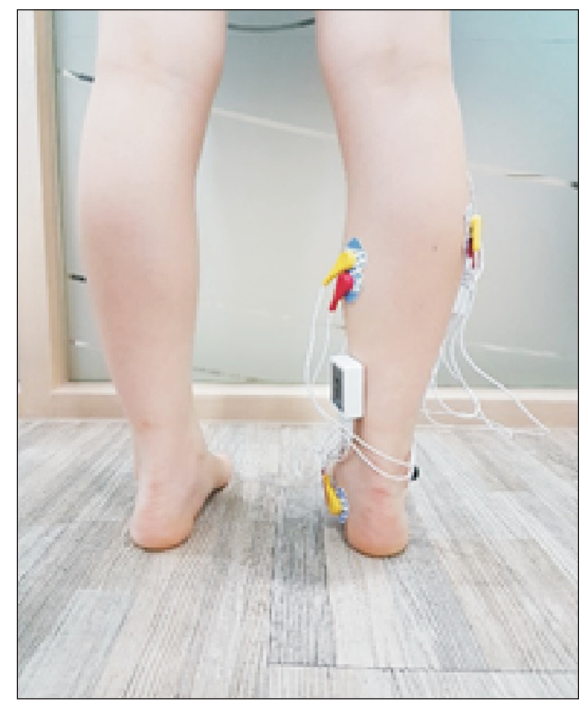

Figure 2. Electromyography (EMG) attachment site: EMG applied to the dominant leg.
NY, USA) was used for data analysis. Independent t-test was used to analyze the characteristics between the flat-feet group and the healthy feet group and the resulting values of the medial and lateral plantar flexion muscle activities (\%MVIC). Statistical significance is set to the value of $\mathrm{p}<0.05$.

\section{RESULTS}

There were no significant differences between two groups in physical characteristics and mean values (Table 1).

There were no significant differences between flat and healthy feet groups in muscle activity of $\mathrm{AH}-$ muscle, TA-muscle, and MG-muscle during BHR-task (Table 2).

The muscle activity of the LG muscle during BHR-task was significantly lower in the flat feet than in the healthy feet group (flat feet: $64.57 \pm 33.74$ [\% MVIC], healthy feet: $90.17 \pm 14.63$ [\% MVIC], $\mathrm{p}=0.038)$.

\section{DISCUSSION}

This study investigated the differences in changes of medial and lateral plantar flexors in subjects with flat feet during BHRtask and analyzed the differences in muscle activity between two groups by measuring the EMG of four medial and lateral plantar flexors. The purpose of this study is to provide basic information to aid in the flat feet intervention methods.

Table 1. Demographics characteristics of participants $(N=21)$

\begin{tabular}{lcccc}
\hline \multicolumn{1}{c}{ Variables } & Flat feet & Healthy feet & $t$ & $\mathrm{p}$ \\
\hline Sex (females) & 11 & 10 & - & - \\
Age (y) & $28.18 \pm 2.56$ & $28.00 \pm 2.78$ & 0.156 & 0.878 \\
Height (cm) & $161.27 \pm 6.63$ & $160.55 \pm 6.08$ & 0.259 & 0.798 \\
Mass (kg) & $60.36 \pm 12.46$ & $59.30 \pm 11.04$ & 0.206 & 0.839 \\
\hline
\end{tabular}

Values are presented as number only or mean \pm standard deviation. -, not available.

Table 2. Medial and lateral plantar flexor activation during heel-raising in each group (unit: \%MVIC)

\begin{tabular}{lcccc}
\hline \multicolumn{1}{c}{ Variables } & Flat feet & Healthy feet & $\mathrm{t}$ & $\mathrm{p}$ \\
\hline Abductor hallucis & $63.99 \pm 19.68$ & $52.76 \pm 24.13$ & 1.17 & 0.255 \\
Tibialis anterior & $29.57 \pm 22.04$ & $29.57 \pm 18.93$ & 0.000 & $>0.999$ \\
Lateral & $64.57 \pm 33.74$ & $90.17 \pm 14.63$ & -2.29 & $0.038^{*}$ \\
$\quad$ & & & \\
$\quad$ gastrocnemius & & & 0.527 & 0.863 \\
$\quad$ gastrocnemius & $88.41 \pm 11.23$ & $85.84 \pm 11.10$ & & \\
\hline
\end{tabular}

$\% \mathrm{MVIC}, \%$ maximum voluntary isometric contraction. *Statistically significant at the level of $p<0.05$. 
It was found in this study that subjects with flat feet have lower LG-muscle activity compared to subjects with normal one, and accordingly, a difference was observed in the use of LG-muscles in performing BHR.

For the difference between groups, while there were no significant differences in AH-muscle, TA-muscle, and MG-muscle, the muscle activity of LG-muscle was statistically significantly low in flat feet group (64.57\% MVIC) compared to Healthy feet group (90.17\% MVIC). A previous study comparing tension of the lower extremity muscles of subjects with flat and Healthy feet reported that the tension of MG-muscle is higher in subjects with flat feet compared to ones with normal one [18]. The result of this study is consistent with a previous one that explained the muscle activity between two groups using the lower biomechanical function to absorb the external shocks in daily life of individuals with flat feet compared to those with normal one [19]. It is considered that this is also applied to the results of this study that the muscle activity of LG-muscle is lower in flat feet group than that of normal group.

The subjects of this study were asked to perform BHR-task. This movement that use ankle plantar flexors applying body weight as a load is a standard clinical measure used by Lovett in early 1900s to measure muscle strength [20]. In order to reduce the influence of the variable, a stable balance was provided by presenting the bipedal heel rise-task. This study, accordingly, the muscle activity was measured by adopting BHR with established reliability to compare the muscle activity of the medial and lateral plantar flexors in subjects with flat feet. The BHR, a commonly performed movement during daily life or sports activity, increases especially the muscle activity of the gastrocnemius muscle. The previous studies have shown that the changes in feet posture may lead to risk of injury to the lower leg in abnormal muscle activity [11].

The significant difference between two groups in this study was found only in LG-muscle. The AH-muscle is one that, together with the TA-muscle, supports the MLA and supports the arch of the feet [9]. In the subjects with flat feet where excessive pronation of the feet occurs due to the collapse of the MLA resulting from insufficient support for the structures during the weight-bearing performance [4], low muscle activities of AH-muscle and TA-muscle are observed. However, the difference in the average muscle activity of the $\mathrm{AH}$-muscle between the two groups during BHR-task was 11.23\% MVIC, though it was not statistically significant (flat feet group: 63.99\% MVIC;
Healthy feet group: $52.76 \%$ MVIC). This is thought to be the factor that activated the $\mathrm{AH}-$ muscle by acting as an appropriate load on the subjects with flat feet in the BHR-task.

It was considered, based on the results of this study, that the subjects with flat feet had difficulties in using MG and LG muscle in balance unlike those with Healthy feet and that activation of their AH-muscle is promoted. Taken together, it is considered that the development of an intervention to promote the activation of LG-muscle and relieve the MG-muscle of individuals with flat feet is needed. The BHR-task was adopted to measure muscle activity of the medial and lateral plantar flexors.

The reason there were difference, though not being the significant one, in muscle activity and their ratio of $\mathrm{AH}$-muscle between flat and Healthy feet groups is that the task acts as a proper loading enough to strongly activates the AH-muscle.

This study has following limitations: First, it is difficult to generalize the results because the total number of subjects was not enough, with 11 and 10 for the flat and Healthy feet group, respectively. In addition, it is also difficult to generalize the results to both male and female because all subjects were female. Second, the muscle activities were analyzed with only one task of BHR, making impossible to compare with other motions and the EMG of only four muscles of the lower extremity was measured rather than including other muscles. Lastly, the subjects just with difference more than $10 \mathrm{~mm}$ in NDT were included in flat feet group and the difference in muscle activity through more detail classification within the flat feet group was not analyzed.

It is recommended, based on the characteristics of medial and lateral plantar flexor in individuals with flat feet observed in this study, to perform studies in future on the intervention considering the biomechanical characteristics of them after supplementing above limitations.

\section{CONCLUSIONS}

This study aimed to investigate the difference in medial and lateral plantar flexors during heel lifting task between flat $(\mathrm{n}=$ 11) and normal $(n=10)$ feet groups and, for the purpose, the EMG of four medial and lateral plantar flexors were measured and their differences were compared and analyzed.

The result showed that the subjects with flat feet have significantly lower activity of LG muscle compared to those of 
subjects with normal one. This indicates that the development of intervention considering the biomechanical characteristics of individuals with flat feet is feasible.

\section{CONFLICTS OF INTEREST}

No potential conflict of interest relevant to this article was reported.

\section{AUTHOR CONTRIBUTIONS}

Conceptualization: WY. Data curation: HJ. Formal analysis: HJ. Funding acquisition: HJ. Investigation: HJ. Methodology: WY. Project administration: WY. Resources: HJ. Software: HJ. Supervision: WY. Validation: WY. Visualization: HJ. Writing original draft: HJ. Writing - review \& editing: WY.

\section{ORCID}

Hui-won Jung, https://orcid.org/0000-0002-7579-3696

\section{REFERENCES}

1. Richie DH Jr. Biomechanics and clinical analysis of the adult acquired flatfoot. Clin Podiatr Med Surg 2007;24(4):617-44, vii.

2. Neumann DA. Ankle and foot. In: Neumann DA editor. Kinesiology of the musculoskeletal system: foundations for rehabilitation. 2nd ed. St. Louis: Mosby; 2009.

3. Fiolkowski P, Brunt D, Bishop M, Woo R, Horodyski M. Intrinsic pedal musculature support of the medial longitudinal arch: an electromyography study. J Foot Ankle Surg 2003;42(6):32733.

4. Bishop C, Arnold JB, May T. Effects of taping and orthoses on foot biomechanics in adults with flat-arched feet. Med Sci Sports Exerc 2016;48(4):689-96.

5. Subotnick SI. The biomechanics of running. Implications for the prevention of foot injuries. Sports Med 1985;2(2):144-53.

6. Magee DJ. Orthopedic physical assessment. 5th ed. St. Louis: W.B. Saunders; 2008.

7. Park MC. The effect of low-dye taping on muscle activity during single-leg standing in people with flatfoot. J Korean Soc
Phys Med 2013;8(4):533-8.

8. Hong Y, Li JX, Fong DT. Effect of prolonged walking with backpack loads on trunk muscle activity and fatigue in children. J Electromyogr Kinesiol 2008;18(6):990-6.

9. Murley GS, Menz HB, Landorf KB. Foot posture influences the electromyographic activity of selected lower limb muscles during gait. J Foot Ankle Res 2009;2:35.

10. Riemann BL, Limbaugh GK, Eitner JD, LeFavi RG. Medial and lateral gastrocnemius activation differences during heel-raise exercise with three different foot positions. J Strength Cond Res 2011;25(3):634-9.

11. Hunt AE, Smith RM. Mechanics and control of the flat versus normal foot during the stance phase of walking. Clin Biomech (Bristol, Avon) 2004;19(4):391-7.

12. Thordarson DB, Schmotzer H, Chon J, Peters J. Dynamic support of the human longitudinal arch. A biomechanical evaluation. Clin Orthop Relat Res 1995;(316):165-72.

13. Cibulka M, Wenthe A, Boyle Z, Callier D, Schwerdt A, Jarman $\mathrm{D}$, et al. Variation in medial and lateral gastrocnemius muscle activity with foot position. Int J Sports Phys Ther 2017;12(2): 233-41.

14. Lange B, Chipchase L, Evans A. The effect of low-Dye taping on plantar pressures, during gait, in subjects with navicular drop exceeding 10 mm. J Orthop Sports Phys Ther 2004; 34(4):201-9.

15. Hermens HJ, Freriks B, Disselhorst-Klug C, Rau G. Development of recommendations for SEMG sensors and sensor placement procedures. J Electromyogr Kinesiol 2000;10(5):361-74.

16. Arinci Incel N, Genç H, Erdem HR, Yorgancioglu ZR. Muscle imbalance in hallux valgus: an electromyographic study. Am J Phys Med Rehabil 2003;82(5):345-9.

17. Criswell E. Cram's introduction to surface electromyography. 2nd ed. Sudbury: Jones \& Bartlett; 2011;371-3.

18. Kendall FP, McCreary EK, Provance PG, Rodgers M, Romani W. Muscles: testing and function with posture and pain. 5th ed. Baltimore: Lippincott Williams \& Wilkins; 2005;400.

19. Lee JE, Park GH, Lee YS, Kim MK. A comparison of muscle activities in the lower extremity between flat and normal feet during one-leg standing. J Phys Ther Sci 2013;25(9):105961.

20. Daniels L, Worthingham C. Muscle testing, techniques of manual examination. Am J Phys Med 1974;53(5):241. 